Диабетическая болезнь почек (ДБП) - серьезное микрососудистое осложнение сахарного диабета (СД), также являющееся основной причиной терминальной стадии почечной недостаточности и ассоциированное с повышенным риском сердечно-сосудистых заболеваний и смертности. Несмотря на достижения в поддержании оптимальных показателей гликемии и артериального давления, приблизительно у 20-40\% пациентов с СД развивается ДБП. Тщательный контроль гликемии и АД замедляет снижение расчетной скорости клубочковой фильтрации и альбуминурии, что позволяет отсрочить дебют и развитие диабетической нефропатии. Препараты инкретинового ряда, такие как агонисты рецептора глюкагоноподобного пептида-1 (ГПП-1) и ингибиторы дипептидилпептидазы-4 (ДПП-4), широко используются в качестве сахароснижающей терапии и демонстрируют улучшение почечных исходов при ДБП. В данном обзоре обсуждаются негликемические свойства препаратов инкретинового ряда и их нефропротективное действие на компоненты метаболического синдрома: ожирение, артериальную гипертензию и дислипидемию; уменьшение оксидативного стресса и воспаление; увеличение натрийуреза.

КЛЮЧЕВЫЕ СЛОВА: сахарный диабет 2 типа; диабетическая болезнь почек; диабетическая нефропатия; инкретины; ингибиторы ДПП-4; агонисты рецептора ГПП-1

\title{
ROLE OF INCRETIN BASED THERAPIES IN THE TREATMENT OF DIABETIC KIDNEY DISEASE
}

\author{
(c) Paola Fioretto*, Andrea Frascati
}

Department of Medicine, University of Padova, Italy

Diabetic kidney disease (DKD), a serious microvascular complication of diabetes mellitus is a leading cause of end-stage renal disease and is associated with an increased risk of cardiovascular morbidity and mortality. Despite advancements in blood glucose and blood pressure (BP) control, $20 \%$ to $40 \%$ of patients with diabetes mellitus develop DKD. Intensive glycaemic and BP control positively influence decline in estimated glomerular filtration rate and albuminuria, thereby delaying the onset and progression of diabetic nephropathy. Incretin based therapies namely glucagon-like peptide-1 receptor agonists (GLP-1 RAs) and dipeptidyl peptidase-4 (DPP-4) inhibitors are widely used glucose lowering agents and have shown favorable renal outcomes in DKD. This article discusses the extra-glycaemic properties of incretin based therapies and their renoprotective effects on components of the metabolic syndrome, including obesity, hypertension and dyslipidaemia; reduction in oxidative stress and inflammation; and increase in natriuresis.

KEYWORDS: type 2 diabetes mellitus; diabetic kidney disease; diabetic nephropathy; incretins; DPP-4 inhibitors; GLP-1 receptor agonists

Диабетическая болезнь почек (ДБП) - серьезное микрососудистое осложнение сахарного диабета (СД), являющееся ведущей причиной терминальной стадии хронической болезни почек (тХБП). Данное состояние характеризуется прогрессирующим снижением функции почек, что приводит к увеличению альбуминурии (>300 мг/сут или >200 нг/мин), снижению скорости клубочковой фильтрации (СКФ), повышению артериального давления (АД), повышению сердечно-сосудистой заболеваемости и смертности по причине сердечно-сосудистых осложнений [1].

\section{ЕСТЕСТВЕННОЕ ТЕЧЕНИЕ ДИАБЕТИЧЕСКОЙ НЕФРОПАТИИ}

Патофизиология ДБП - сложный и многофакторный процесс, в котором задействованы как генетические факторы, так и факторы окружающей среды [2]. На момент постановки диагноза приблизительно у 70\% пациентов с сахарным диабетом 1 типа (СД1) и у 50\% пациентов с са- харным диабетом 2 типа (СД2) наблюдается повышение СКФ на 25-50\%, сопровождающееся тубулярной гиперплазией, гипертрофией и увеличением размеров почек. Хотя вышеуказанные признаки клубочковой гиперфильтрации ясно манифестируют при СД1, у пациентов с СД2 симптомы зависят от возраста, длительности диабета, компенсации углеводного обмена и контроля АД [2, 3].

Несмотря на осведомленность о факторах риска и успехи в медикаментозной компенсации повышения АД, углеводного и липидного обменов, приблизительно у $20-40 \%$ пациентов с СД развивается ДБП [1, 2]. Около 3\% пациентов с впервые выявленным СД2 имеют явную ДБП [3]. При СД2, помимо хронической гипергликемии, развитию ДБП предшествуют долгосрочные факторы сердечно-сосудистого риска, такие как ожирение, артериальная гипертензия (АГ) и дислипидемия [2, 4]. На протяжении десятилетий отмечается снижение распространенности диабет-ассоциированных осложнений, таких как инсульт, острый инфаркт миокарда, ампутации и летальность, связанная с гипергликемическими комами. Однако вместе 
Факторы риска развития диабетической болезни почек
Эффекты терапии препаратами инкретинового ряда

\begin{abstract}
Пациенты
с диабетической

болезнью почек
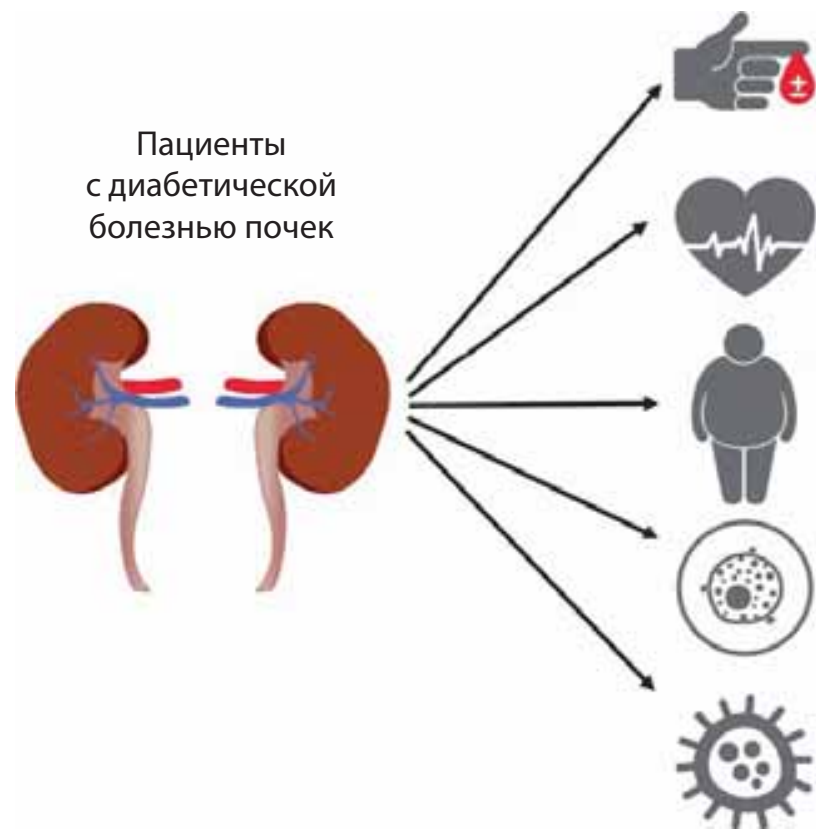

с этим снижение распространенности ДБП за это же время невелико [5]. Поэтому, с учетом тяжести ДБП, существует необходимость в эффективной тактике ведения, сосредоточенной на многоплановом подходе, для предотвращения и замедления прогрессии заболевания. В дополнение к показателям используемым в настоящее время - расчетная СКФ, соотношение альбумин/креатинин, - существует необходимость в других надежных биомаркерах плазмы крови и мочи, которые позволят определить группы риска прогрессирующей ДБП и улучшат клинические исходы за счет индивидуализированного подхода к лечению [6].
\end{abstract}
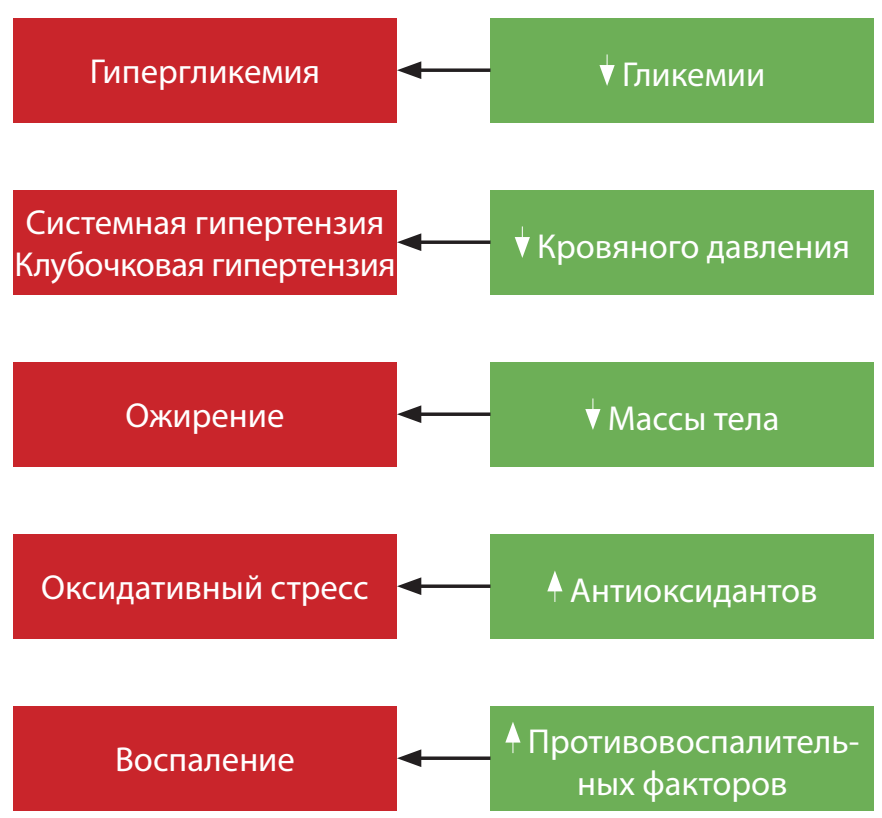

Рис. 1. Нефропротективное действие препаратов инкретинового ряда.

\section{ЛЕЧЕНИЕ ДИАБЕТИЧЕСКОЙ БОЛЕЗНИ ПОЧЕК}

Строгий контроль гликемии и АД замедляет снижение СКФ и положительно влияет на альбуминурию, таким образом позволяя отсрочить дебют и прогрессирование ДБП [7]. Долгосрочные исследования среди пациентов с СД2, такие как UKPDS и ADVANCE, показали, что тщательный контроль гликемии приводит к устойчивому снижению почечных осложнений $[8,9]$.

Результаты исследования UKPDS показали, что тщательный контроль гликемии и АД у пациентов с СД2 приводил к снижению относительного риска развития микроальбуминурии (на 33\%) и микрососудистых исходов (на 25\%) через 12 лет [8, 10]. Польза интенсивного контроля гликемии в течение 5 лет была показана в результатах исследования ADVANCE: снижение риска развития терминальной ХБП на 65\% [9]. Кроме того, по результатам длительного (22 года) исследования DCCT-EDIC в группе интенсивного лечения пациентов с СД1 риск снижения СКФ уменьшился на $50 \%$ [11].

Влияние препаратов инкретинового ряда на почечные факторы риска

Хроническая гипергликемия, артериальная гипертензия и дислипидемия приводят к гемодинамическим изменениям, которые воздействуют на внутриклеточные сигнальные пути, транскрипционные факторы, цитокины, хемокины и ростовые факторы, приводящие к развитию и прогрессированию ДБП [12]. Препараты инкретинового ряда - агонисты глюкагоноподобного пептида-1 (аГПП-1) и ингибиторы дипептидилпептидазы-4 (иДПП-4) показали положительный эффект на почечные исходы при ДБП. В дополнение к контролю гликемии, аГПП-1 и иДПП-4 имеют расширенный нефропротективный эффект, так как они влияют на компоненты метаболического синдрома: ожирение, АГ и дислипидемию, снижают оксидативный стресс и воспаление, увеличивают натрийурез (рис. 1) [2].

\section{Нефропротективное действие агонистов}

глюкагоноподобного пептида-1

У здоровых людей инфузия ГПП-1 заметно повышала экскрецию натрия с мочой, хотя достоверных изменений в концентрациях предсердного натрийуретического пептида или натрийуретического пептида типа В не отмечалось [13]. Подобным образом, в рандомизированном двойном слепом плацебо-контролируемом исследовании у пациентов с СД2 с избыточной массой тела и нормальной почечной функцией (СКФ $\geq 60$ мл/мин/1,73 м²) лечение эксенатидом увеличивало экскрецию натрия в проксимальных канальцах, однако не воздействовало на СКФ, почечный кровоток и клубочковое давление [14].

Исследование с малым периодом наблюдения продемонстрировало, что лираглутид снижал реабсорбцию натрия в проксимальных канальцах без изменения почечной гемодинамики [15]. Помимо этого, после лечения лираглутидом пациентов с СД2, персистирующей альбуминурией (альбумин/креатинин мочи >30 мг/г) и СКФ $\geq 30$ мл/мин/1,73 м² в течение 12 нед отмечалось клинически значимое снижение экскреции альбумина с мочой на 32\% ( $p=0,017)$. Также отмечалось снижение активности ренина плазмы (35\%, $p=0,060)$, средней концентрации ренина плазмы $(37 \%, \mathrm{p}=0,030)$ и ангиотензина II 
(43\%, p=0,022), что указывает на роль лираглутида в ингибировании ренин-ангиотензиновой системы (РАС) [16].

После лечения пациентов с СД2 и микроальбуминурией эксенатидом отмечалось значительное снижение экскреции альбумина, трансформирующего фактора роста- $\beta 1$ и коллагена IV типа, а также снижение массы тела и систолического АД, что свидетельствует о нефропротективных свойствах препарата, помимо непосредственного влияния на контроль гликемии [17]. По результатам 52-недельного исследования DURATION-2 у пациентов с СД2, получавших эксенатид во время исследования, отмечалось снижение гликированного гемоглобина $\left(\mathrm{HbA}_{1 c}\right)$, массы тела, АД, а также значительное снижение кардиоваскулярных маркеров, таких как соотношение альбумин/креатинин (34\%, $\mathrm{p}<0,05)$, в сравнении с пациентами, которых перевели на ситаглиптин $(18 \%, \mathrm{p}<0,05)$ и пиоглитазон $(23 \%, \mathrm{p}<0,05)[18]$.

У пациентов с СД2 и высоким сердечно-сосудистым риском встречаемость почечных микрососудистых событий (включая макроальбуминурию, удвоение сывороточного креатинина, ТХБП и почечную смерть) была ниже в группе лираглутида по сравнению с группой плацебо (отношение рисков [OP] 0,78; 95\% доверительный интервал [ДИ] 0,67-0,92; $p=0,003$ ) по данным исследования LEADER [19]. У меньшего количества пациентов группы лираглутида отмечался дебют персистирующей альбуминурии (ОР 0,74; 95\% ДИ 0,60-0,91; p=0,004) [20]. Сходные результаты по почечным исходам наблюдались в исследовании EMPA-REG OUTCOME, где применялся препарат из группы ингибиторов натрийглюкозного котранспортера-2 для лечения пациентов с СД2 и СКФ $\geq 30$ мл/мин/1,73 м². У пациентов, получавших лечение эмпаглифлозином, отмечались более медленное прогрессирование болезни почек (ОР 0,61; 95\% ДИ 0,53-0,70; p<0,001) и более низкая встречаемость почечных событий по сравнению с пациентами из группы плацебо [21].

Другие нефропротективные свойства агонистов рецептора ГПП-1, такие как снижение оксидативного стресса и противовоспалительный эффект, были показаны на животных моделях [22, 23]. Лечение лираглутидом уменьшало прогрессию нефропатии у мышей линии KK/Ta-Akita путем уменьшения альбуминурии и мезангиальной экспансии, снижения уровней гломерулярного супероксида и почечной оксидазы никотинамидадениндинуклеотидфосфата (NADPH) и повышения активности почечного циклического аденозинмонофосфата АМФ (цАМФ) и протеинкиназы А (PKA). В присутствии ингибиторов аденилатциклазы и РКА данных явлений не наблюдалось. Эти данные свидетельствуют о том, что ГПП-1 играет ключевую роль в защите от повышенного почечного оксидативного стресса при гипергликемии за счет ингибирования NADPH-оксидазы и активации сигнального пути цАМФ-РКА [22]. В исследованиях на мышах со стрептозотоцин-индуцированным СД1 эксендин-4 оказывает противовоспалительное действие, непосредственно воздействуя на рецепторы ГПП-1 и подавляя продукцию провоспалительных цитокинов и молекулы межклеточной адгезии-1 [23].

Нефропротективное действие ингибиторов ДПП-4

У пациентов с СД2 при кратковременном (1 мес) лечении ситаглиптином ингибирование ДПП-4 приводит к увеличению натрийуреза: блокируется реабсорбция натрия в дистальных извитых канальцах без воздействия на меха- низмы почечной гемодинамики [24]. В отличие от иДПП-4, аГПП-1 и ингибиторы SGLT-2 действуют на уровне проксимальных канальцев.

Исследования показали, что фермент ДПП-4 имеет множество субстратов, помимо ГПП-1 и гастроингибирующего пептида (ГИП). Фактор стромальных клеток-1a (SDF-1a), хемокин, широко экспрессирующийся в клетках дистальных канальцев, является физиологическим субстратом ДПП-4. Лечение ситаглиптином приводит к увеличению концентрации интактного SDF-1 в в плазме, данный хемокин в доклинических исследованиях показал натрийуретическую активность [24].

Лечение линаглиптином в комбинации с ингибиторами ренин-ангиотензин-альдостероновой системы (РАAС) приводило к значительному снижению альбуминурии (32\%; 95\% ДИ 42-21; р<0,05) у пациентов с СД2 с почечными нарушениями (соотношение альбумин/креатинин в моче 30-3000 мг/г) после 24 нед лечения. Эти данные свидетельствуют о взаимодополняющем влиянии на снижение микроальбуминурии при сочетании ингибиторов PAAC и ДПП-4 [25]. В исследовании SAVOR-TIMI саксаглиптин также уменьшал соотношение альбумин-креатинин в большой гетерогенной популяции пациентов с СД2 с нормо-, микрои макроальбуминурией, независимо от исходной СКФ [26].

Другие субстраты фермента ДПП-4, такие как предсердный натрийуретический пептид, субстанция Р, субъединица $\beta$ меприна А и нейропептид Ү также обладают противовоспалительными, натрийуретическими и вазоактивными свойствами, что указывает на независимость вышеописанного действия ДПП-4 от ГПП-1 [2].

\section{ЗАКЛЮЧЕНИЕ}

Лечение ДБП - сложная задача, так как заболевание имеет прогрессирующую природу и вовлекает множество патофизиологических механизмов. Многофакторный подход, включающий поддержание оптимальных показателей гликемии, АД и липидного профиля, а также блокирование РАС, является эффективным для улучшения почечных и сердечно-сосудистых исходов. Тем не менее необходимы дальнейшие исследования патофизиологических механизмов, связанных с прогрессированием ДБП, чтобы определить новые цели для лечения. Согласно исследованиям на животных моделях, препараты инкретинового ряда, такие как аГПП-1 и иДПП-4, обеспечивают нефропротекцию и замедление прогрессии ДБП за счет ингибирования воспаления и оксидативного стресса. аГПП-1, в дополнение к снижению $\mathrm{HbA}_{1 c^{\prime}}$ снижают АД и массу тела, увеличивают натрийурез. Кроме того, иДПП-4 имеют положительное влияние на альбуминурию. Однако большинство исследований по почечной функции и клиническим почечным исходам, скорее представляют собой данные по безопасности, нежели конечные точки эффективности, что подчеркивает необходимость дальнейших исследований с конечными точками, сосредоточенными на почечных аспектах.

\section{ДОПОЛНИТЕЛЬНАЯ ИНФОРМАЦИЯ}

Источники финансирования. Публикация статьи на русском и английском языках поддержана компанией «Новартис». Спонсор не принимал участие в проведении поисково-аналитической работы, анализе и интерпретации данных. 
Конфликт интересов. P. Fioretto получала гонорары за лекции и оплату за консультации от Astra Zeneca, Boerhinger Ingelheim, Eli Lilly, Novartis. A. Frascati не имеет явных и потенциальных конфликтов интереса, связанных с публикацией данной статьи. Эта статья является резюме лекции Paola Fioretto, представленной на научной конференции Novartis 11 июля 2017 года в Москве.

Участие авторов. Авторы соответствуют критериям International Committee of Medical Journal Editors (ICMJE) авторства данной статьи, отвечают за целостность работы и утвердили итоговую версию статьи к публикации.

Благодарности. Авторы выражают благодарность д.м.н. Nihal Ganesh Maremanda и д.м.н. Lakshmi Deepa G., сотрудникам Novartis Healthcare Private Limited (Индия) за помощь в подготовке рукописи, в соответствии с рекомендациями по надлежащей публикационной практике (good publication practice (GPP3), http://www.ismpp.org/gpp3).

\section{СПИСОК ЛИТЕРАТУРЫ | REFERENCES}

1. Rossing P, Persson F, Frimodt-Moller M. Prognosis and treatment of diabetic nephropathy: Recent advances and perspectives. Nephrol Ther. 2018;14 Suppl 1:S31-S37. doi: 10.1016/j.nephro.2018.02.007

2. Muskiet MH, Smits MM, Morsink LM, Diamant M. The gut-renal axis: do incretin-based agents confer renoprotection in diabetes? Nat Rev Nephrol. 2014;10(2):88-103. doi: 10.1038/nrneph.2013.272

3. Gheith O, Farouk N, Nampoory N, et al. Diabetic kidney disease: world wide difference of prevalence and risk factors. J Nephropharmacol. 2016;5(1):49-56.

4. United States Renal Data System. 2014 USRDS annual data report: Epidemiology of kidney disease in the United States. Bethesda: National Institutes of Health, National Institute of Diabetes and Digestive and Kidney Diseases; 2014

5. Gregg EW, Li Y, Wang J, et al. Changes in diabetes-related complications in the United States, 1990-2010. N Engl J Med. 2014;370(16):1514-1523. doi: 10.1056/NEJMoa1310799

6. Hanssen NM, Russell N, Cooper ME. Recent advances in glucose-lowering treatment to reduce diabetic kidney disease. Expert Opin Pharmacother. 2015;16(9):1325-1333. doi: 10.1517/14656566.2015.1041502

7. Kim Y, Park CW. New therapeutic agents in diabetic nephropathy Korean J Intern Med. 2017;32(1):11-25. doi: 10.3904/kjim.2016.174

8. Bilous R. Microvascular disease: what does the UKPDS tell us about diabetic nephropathy? Diabet Med. 2008;25 Suppl 2:25-29. doi: 10.1111/j.1464-5491.2008.02496.x

9. Perkovic V, Heerspink HL, Chalmers J, et al. Intensive glucose control improves kidney outcomes in patients with type 2 diabetes. Kidney Int. 2013;83(3):517-523. doi: 10.1038/ki.2012.401

10. Holman RR, Paul SK, Bethel MA, et al. 10-year follow-up of intensive glucose control in type 2 diabetes. N Engl J Med. 2008;359(15):1577-1589. doi: 10.1056/NEJMoa0806470

11. The DCCT/EDIC Research Group, de Boer IH, Sun W, et al. Intensive diabetes therapy and glomerular filtration rate in type 1 diabetes. $N$ Engl J Med. 2011;365(25):2366-2376. doi: 10.1056/NEJMoa1111732

12. Badal SS, Danesh FR. New insights into molecular mechanisms of diabetic kidney disease. Am J Kidney Dis. 2014;63(2 Suppl 2):S63-83. doi: 10.1053/j.ajkd.2013.10.047

13. Skov J, Holst JJ, Gotze JP, et al. Glucagon-like peptide-1: effect on pro-atrial natriuretic peptide in healthy males. Endocr Connect. 2014;3(1):11-16. doi: 10.1530/EC-13-0087

14. Tonneijck L, Smits MM, Muskiet MHA, et al. Acute renal effects of the GLP-1 receptor agonist exenatide in overweight type 2 diabetes patients: a randomised, double-blind, placebo-controlled trial. Diabe tologia. 2016;59(7):1412-1421. doi: 10.1007/s00125-016-3938-z
15. Skov J, Pedersen M, Holst JJ, et al. Short-term effects of liraglutide on kidney function and vasoactive hormones in type 2 diabetes: a randomized clinical trial. Diabetes Obes Metab. 2016;18(6):581-589. doi: 10.1111/dom.12651

16. von Scholten BJ, Persson F, Rosenlund S, et al. The effect of liraglutide on renal function: A randomized clinical trial. Diabetes Obes Metab. 2017:19(2):239-247. doi: 10.1111/dom.12808

17. Zhang $H$, Zhang $X, H u C$, Lu W. Exenatide reduces urinary transforming growth factor-beta1 and type IV collagen excretion in patients with type 2 diabetes and microalbuminuria. Kidney Blood Press Res. 2012;35(6):483-488. doi: 10.1159/000337929

18. Wysham C, Bergenstal R, Malloy J, et al. DURATION-2: efficacy and safety of switching from maximum daily sitagliptin or pioglitazone to once-weekly exenatide. Diabet Med. 2011;28(6):705-714. doi: 10.1111/j.1464-5491.2011.03301.x

19. Marso SP, Daniels GH, Brown-Frandsen K, et al. Liraglutide and Cardiovascular Outcomes in Type 2 Diabetes. N Engl J Med. 2016;375(4):311-322. doi: 10.1056/NEJMoa1603827

20. Mann JFE, Orsted DD, Brown-Frandsen K, et al. Liraglutide and Renal Outcomes in Type 2 Diabetes. N Engl J Med. 2017;377(9):839-848. doi: 10.1056/NEJMoa1616011

21. Wanner C, Inzucchi SE, Lachin JM, et al. Empagliflozin and Progression of Kidney Disease in Type 2 Diabetes. N Engl J Med. 2016;375(4):323-334. doi: 10.1056/NEJMoa1515920

22. Fujita H, Morii T, Fujishima H, et al. The protective roles of GLP-1R sig naling in diabetic nephropathy: possible mechanism and therapeutic potential. Kidney Int. 2014;85(3):579-589. doi: 10.1038/ki.2013.427

23. Kodera R, Shikata K, Kataoka HU, et al. Glucagon-like peptide-1 receptor agonist ameliorates renal injury through its anti-inflammatory action without lowering blood glucose level in a rat model of type 1 diabetes. Diabetologia. 2011;54(4):965-978. doi: 10.1007/s00125-010-2028-X

24. Lovshin JA, Rajasekeran H, Lytvyn Y, et al. Dipeptidyl Peptidase 4 Inhibition Stimulates Distal Tubular Natriuresis and Increases in Circulating SDF-1alpha(1-67) in Patients With Type 2 Diabetes. Diabetes Care. 2017;40(8):1073-1081. doi: 10.2337/dc17-0061

25. Groop PH, Cooper ME, Perkovic V, et al. Linagliptin lowers albuminuria on top of recommended standard treatment in patients with type 2 diabetes and renal dysfunction. Diabetes Care. 2013;36(11):3460-3468. doi: 10.2337/dc13-0323

26. Mosenzon O, Leibowitz G, Bhatt DL, et al. Effect of Saxagliptin on Renal Outcomes in the SAVOR-TIMI 53 Trial. Diabetes Care. 2017:40(1):69-76. doi: 10.2337/dc16-0621

\section{ИНФОРМАЦИЯ ОБ АВТОРАХ [AUTHORS INFO]}

*Paola Fioretto, MD, PhD, Department of Medicine; address: Via Giustiniani 2, 35128 Padova, Italy; ORCID: http://orcid.org/0000-0003-3445-0387; e-mail: paola.fioretto@unipd.it

Andrea Frascati, MD, Department of Medicine; e-mail: andrea.frascati@unipd.it

\section{ЦИТИРОВАТЬ:}

Fioretto P, Frascati A. Роль препаратов инкретинового ряда в лечении диабетической нефропатии // Сахарный диабет. — 2018. — T. 21. — №5. — C. 395-398. doi: 10.14341/DM9845

\section{TO CITE THIS ARTICLE:}

Fioretto P, Frascati A. Role of incretin based therapies in the treatment of diabetic kidney disease. Diabetes Mellitus. 2018;21(5):395-398. doi: 10.14341/DM9845 